\title{
Video Article \\ Measurement of Natural Killer Cell-Mediated Cytotoxicity and Migration in the Context of Hepatic Tumor Cells
}

\author{
Suresh Chava ${ }^{1}$, Suresh Bugide ${ }^{1}$, Romi Gupta ${ }^{1}$, Narendra Wajapeyee ${ }^{1}$ \\ ${ }^{1}$ Department of Biochemistry and Molecular Genetics, University of Alabama at Birmingham
}

Correspondence to: Narendra Wajapeyee at nwajapey@uab.edu

URL: https://www.jove.com/video/60714

DOI: doi: $10.3791 / 60714$

Keywords: Medicine, Issue 156, NK cells, cancer, migration, cytotoxicity, transcription factors, flow cytometry

Date Published: 2/22/2020

Citation: Chava, S., Bugide, S., Gupta, R., Wajapeyee, N. Measurement of Natural Killer Cell-Mediated Cytotoxicity and Migration in the Context of Hepatic Tumor Cells. J. Vis. Exp. (156), e60714, doi:10.3791/60714 (2020).

\section{Abstract}

Natural killer (NK) cells are a subset of the cytotoxic lymphocyte population of the innate immune system and participate as a first line of defense by clearing pathogen-infected, malignant, and stressed cells. The ability of NK cells to eradicate cancer cells makes them an important tool in the fight against cancer. Several new immune-based therapies are under investigation for cancer treatment which rely either on enhancing NK cell activity or increasing the sensitivity of cancer cells to NK cell-mediated eradication. However, to effectively develop these therapeutic approaches, cost-effective in vitro assays to monitor NK cell-mediated cytotoxicity and migration are also needed. Here, we present two in vitro protocols that can reliably and reproducibly monitor the effect of NK-cell cytotoxicity on cancer cells (or other target cells). These protocols are non-radioactivity-based, simple to set up, and can be scaled up for high-throughput screening. We also present a flow cytometry-based protocol to quantitatively monitor NK cell migration, which can also be scaled up for high-throughput screening. Collectively, these three protocols can be used to monitor key aspects of NK cell activity that are necessary for the cells' ability to eradicate dysfunctional target cells.

\section{Video Link}

The video component of this article can be found at https://www.jove.com/video/60714/

\section{Introduction}

The ability of the human body to identify non-self and eradicate foreign objects is key to human survival against pathogens and malignancies ${ }^{1}$. The human immune response plays the most important role in this process ${ }^{2,3,4}$. Based on key characteristics and functions, the human immune system can be broadly classified into two major functional groups: the adaptive immune system and the innate immune system. The adaptive immune system is typically specific to a given pathogen and has immunological memory and, thus, is long-lasting and responsive to future reinfection by the same pathogen ${ }^{5,6,7,8,9}$. In contrast, innate immunity is much broader in its target eradication and relatively nonspecific. Therefore, typically, the innate immune response serves as the first line of immunological defense ${ }^{10}$. Natural killer (NK) cells belong to the innate immune system and represent $10-15 \%$ of total circulating lymphocytes ${ }^{11}$. NK cells eradicate target cells via two major mechanisms. First, upon binding to target cells expressing activating ligands, NK cells release the membrane-disrupting protein perforin and serine protease granzymes through exocytosis, which jointly induce apoptosis in target cells ${ }^{12,13,14,15}$. Additionally, NK cells expressing FasL and tumor necrosis factor-related apoptosis-inducing ligand (TRAIL) interact with target cells expressing death receptors (Fas/CD95), leading to caspase-dependent apoptosis ${ }^{16}$. Most importantly, NK cells do not require prestimulation, such as antigen presentation, to eradicate pathogen-infected or malignant cells; thus, they are usually in a ready-to-kill state ${ }^{17,18}$. In order to inhibit tumor development and progression and eradicate cancer cells, NK cells must migrate to the tumor site and, once in the tumor microenvironment, identify and attack the target cells.

In the past, NK-cell effector functions were mainly monitored by degranulation and cytotoxicity assays ${ }^{19,20,21}$. NK cell cytotoxicity can also be measured by ${ }^{51}$ chromium release assay ${ }^{22,23,24,25}$. However, this assay has some specific requirements, including the need for a gamma counter, and is radioactivity-based, which requires training in handling and disposal of radioactive materials and poses a level of risk to the user. Therefore, several new non-radioactivity-based assays have been developed and employed to study NK cell activity.

Here, we describe two such protocols, colorimetric lactic dehydrogenase (LDH) measurement-based NK cell-mediated cytotoxicity assay and calcein acetoxymethyl (AM) staining-based microscopic method to measure NK cell-mediated cancer cell eradication. These assays do not require the use of radioisotopes, are straightforward, sensitive, and reproducibly identify factors that modulate NK cell function. Additionally, because NK cell function cannot be fully evaluated without monitoring changes in NK cell migration, we also present a flow cytometry-based quantitative method to monitor NK cell migration. 


\section{Preparation of Culture Medium for NK Cells and Hepatic Tumor Cells}

1. Use human natural killer cells (e.g., NK92MI) and human liver cancer cell line (e.g., SK-HEP-1).

2. Prepare NK cell culture medium for NK92MI human NK cells by adding the following components to 500 mL of minimum essential medium Eagle alpha without ribonucleosides and deoxyribonucleosides to the indicated final concentrations: $0.02 \mathrm{mM}$ folic acid $(100 \mu \mathrm{L}$ of $100 \mathrm{mM}$ folic acid), $0.2 \mathrm{mM}$ myoinositol ( $500 \mu \mathrm{L}$ of $200 \mathrm{mM}$ myoinositol), $0.1 \mathrm{mM} \beta$-mercaptoethanol (3.5 $\mu \mathrm{L}$ of $14.3 \mathrm{M} \beta$-mercaptoethanol), $2 \mathrm{mM} \mathrm{L}$ glutamine (5 mL of $200 \mathrm{mM} \mathrm{L-glutamine),} \%$ penicillin/streptomycin (5 mL of 100x penicillin/streptomycin), 12.5\% fetal bovine serum (FBS), and $12.5 \%$ horse serum. Mix and filter sterilize using a $0.22 \mu \mathrm{m}$ sterile filtration unit, and store at $4{ }^{\circ} \mathrm{C}$.

3. Prepare culture medium for hepatic tumor cell line SK-HEP-1 by adding $10 \%$ FBS and $1 \%$ penicillin/streptomycin to high-glucose Dulbecco's modified Eagle medium (DMEM) containing 2 mM L-glutamine.

\section{Colorimetric LDH Measurement-based NK Cell-mediated Cytotoxicity Assay}

1. Grow SK-HEP-1 cells to $70-80 \%$ confluency in a $100 \mathrm{~mm}$ cell culture Petri dish in $5 \% \mathrm{CO}_{2}$ at $37{ }^{\circ} \mathrm{C}$ in a $\mathrm{CO}_{2}$ incubator. Generate a single-cell suspension by first washing cells with $5 \mathrm{~mL}$ of $1 \times$ phosphate-buffered saline (PBS) followed by incubation with $1 \mathrm{~mL}$ of $0.25 \%$ trypsin-EDTA in $5 \% \mathrm{CO}_{2}$ at $37^{\circ} \mathrm{C}$ in a $\mathrm{CO}_{2}$ incubator until a single cell suspension is generated.

NOTE: NK cell activity can be induced by stressed cancer cells due to changes in expression of NK cell-activating ligand in cancer cells. Therefore, healthy and sub-confluent cancer cells should be used for accurate results. It is also important to note that NK cell receptors and ligands can be sensitive to trypsinization ${ }^{26,27}$. Therefore, the trypsinization protocol should be carefully optimized and excessive trypsinization should be avoided.

2. After trypsinization, add $10 \mathrm{~mL}$ of SK-HEP-1 culture medium and centrifuge at $160 \times \mathrm{g}$ for $3 \mathrm{~min}$ in a $15 \mathrm{~mL}$ sterile conical centrifuge tube. Wash the cell pellet with $5 \mathrm{~mL}$ of $1 \times$ PBS and resuspend in $5 \mathrm{~mL}$ of culture medium.

3. In parallel, centrifuge the NK92MI cells at $160 \times \mathrm{g}$ for $3 \mathrm{~min}$ in a $15 \mathrm{~mL}$ sterile centrifuge tube. Wash the cell pellet with $5 \mathrm{~mL}$ of $1 \times \mathrm{PBS}$ and resuspend in $5 \mathrm{~mL}$ of $\mathrm{NK} 92 \mathrm{MI}$ cell culture medium.

NOTE: NK92MI cells are grown in NK cell culture medium and obtained similarly as described in step 2.1.

4. Count the SK-HEP-1 and NK92MI cells using a hemocytometer or any available automated cell counter.

5. Add SK-HEP-1 cells (target cells) $\left(1 \times 10^{4} / 100 \mu \mathrm{L} /\right.$ well) and NK92MI cells (effector cells) $\left(1 \times 10^{5} / 100 \mu \mathrm{L} /\right.$ well) in the ratio of $1: 10$ target:effector and seed in triplicate wells in a 96 well plate.

6. Incubate the 96 well plate at $37{ }^{\circ} \mathrm{C}$ in an atmosphere of $95 \%$ air and $5 \% \mathrm{CO}_{2}$ for $3 \mathrm{~h}$. After incubation, centrifuge plate at $450 \times \mathrm{g}$ for $5 \mathrm{~min}$ at room temperature.

7. Without disturbing the cell pellet, collect $100 \mu \mathrm{L}$ of supernatant from each well and transfer to a well in a new 96 well plate.

8. Add $50 \mu \mathrm{L}$ of $\mathrm{LDH}$ substrate, mix well, and incubate the plate for $20 \mathrm{~min}$ at room temperature in the dark.

9. Stop the reaction by adding $50 \mu \mathrm{L}$ of stop solution ( $50 \%$ dimethylformamide and $20 \%$ sodium dodecyl sulfate at $\mathrm{pH} 4.7)$. Immediately measure the absorbance of the plate at $490 \mathrm{~nm}$ and $680 \mathrm{~nm}$ using a plate reader.

10. Subtract the absorbance at $680 \mathrm{~nm}$ from the absorbance at $490 \mathrm{~nm}$. Calculate the percent (\%) NK cell cytotoxicity using the formula below. NK cell cytotoxicity $(\%)=\frac{L D H \text { experimental }-L D H \text { ef fector cells }-L D H \text { spontaneous }}{L D H \text { maximal }-L D H \text { spontaneos }} \times 100$

where LDH experimental (effector + target cells) is the absorbance of NK92MI cells and SK-HEP-1 cells, LDH effector cells is the absorbance of NK92MI cells alone, LDH spontaneous is the absorbance of SK-HEP-1 cells alone, and LDH maximal is the absorbance of SK-HEP-1 cells with lysis buffer.

NOTE: To reduce serum interference, always use the following controls: target cells alone (SK-HEP-1), effector cells alone (NK92MI), target cells with lysis buffer as a complete lysis control, target cell medium, NK92MI medium, as well as target cell medium and NK92MI medium in a 1:1 ratio.

\section{Calcein AM Staining-based Microscopic Method for Measuring NK Cell-mediated Cytotoxicity}

1. Culture SK-HEP-1 cells to $70-80 \%$ confluency. Generate a single-cell suspension by first washing cells with $5 \mathrm{~mL}$ of $1 \mathrm{x}$ PBS followed by incubation with $1 \mathrm{~mL}$ of $0.25 \%$ trypsin-EDTA.

2. Centrifuge SK-HEP-1 cells in a $1 \mathrm{~mL}$ sterile centrifuge tube at $160 \mathrm{xg}$ for $3 \mathrm{~min}$. Resuspend the pellet in $3 \mathrm{~mL}$ of serum-free DMEM.

3. Add $1.5 \mu \mathrm{L}$ of calcein AM solution $(10 \mathrm{mM})$ to SK-HEP-1 cells and incubate for $30 \mathrm{~min}$ at room temperature. Centrifuge calcein AM-labeled SK-HEP-1 cells at $160 \times g$ for $3 \mathrm{~min}$ in a $15 \mathrm{~mL}$ sterile centrifuge tube.

4. Wash cells twice with $5 \mathrm{~mL}$ of $1 \times$ PBS to remove excess calcein AM dye.

5. In parallel, centrifuge NK92Ml cells at $160 \times g$ for $3 \mathrm{~min}$ in a $15 \mathrm{~mL}$ sterile centrifuge tube. Wash the cell pellet once with $5 \mathrm{~mL}$ of $1 \times \mathrm{PBS}$ and resuspend in $5 \mathrm{~mL}$ of NK92Ml cell medium.

6. Count calcein AM-labeled SK-HEP-1 cells and NK92MI cells using a hemocytometer or an automated cell counter.

7. Resuspend SK-HEP-1 cells in culture medium at $1 \times 10^{5}$ cells $/ \mathrm{mL}$ and NK92MI cells at $1 \times 10^{6} \mathrm{cells} / \mathrm{mL}$ in NK cell medium.

8. Plate calcein AM-labeled SK-HEP-1 cells (target cells) $\left(1 \times 10^{4} / 100 \mu \mathrm{L} /\right.$ well) with NK92MI cells (effector cells) $\left(1 \times 10^{5} / 100 \mu \mathrm{L} /\right.$ well $)(1: 10$ target:effector ratio) per well in triplicate wells in a 96 well plate.

9. Incubate the 96 well plate at $37^{\circ} \mathrm{C}$ in an atmosphere of $95 \%$ air and $5 \% \mathrm{CO}_{2}$ for $4 \mathrm{~h}$. After incubation, capture fluorescence images of the calcein AM-labeled cells using a fluorescence microscope at 10x magnification. Capture at least 10 different fields of each replicate for each treatment condition.

10. Randomly select 10 images for each replicate and count calcein AM-positive labeled target cells incubated with or without NK92MI cells. Calculate \% cytotoxicity using the formula below. 
NK cell cytotoxicity $(\%)=\frac{\text { Number of target cells co-cultured with } N \text { K } 92 M I \text { cells }}{\text { Number of target cells cultured without } N K 92 M I \text { cells }} \times 100$

NOTE: As controls, use target cells without NK92MI cells and completely lysed target cells as complete lysis control. For complete lysis, incubate the cells in $0.5 \%$ Triton X-100 for $1 \mathrm{~h}(20 \mu \mathrm{L}$ of $5 \%$ Triton X-100 in $200 \mu \mathrm{L}$ of culture media).

\section{NK Cell Migration Assay}

1. Grow NK92MI cells and centrifuge cells at $160 \times g$ for $3 \mathrm{~min}$ in a $15 \mathrm{~mL}$ sterile centrifuge tube.

2. Wash the cell pellet twice with $5 \mathrm{~mL}$ of $1 \times$ PBS and resuspend the cells in $3 \mathrm{~mL}$ of serum-free NK92Ml cell medium. Count NK92MI cells using a hemocytometer or an automated cell counter.

3. Plate NK92MI cells $\left(2.5 \times 10^{5}\right.$ cells $/ 100 \mu \mathrm{L} /$ well $)$ in the upper compartment of transwell permeable chamber $(6.5 \mathrm{~mm}$ diameter insert and $5 \mu \mathrm{m}$ pore size).

4. In the bottom chamber add $0.6 \mathrm{~mL}$ of serum-free medium containing material to be tested for NK cell chemoattractant properties (e.g., conditioned medium, chemokines, cytokines)

NOTE: When preparing conditioned medium, use reduced-serum medium without added serum to eliminate interference from serum proteins in the migration assay.

5. Incubate the 24 well permeable chambers at $37^{\circ} \mathrm{C}$ for $4 \mathrm{~h}$. After $4 \mathrm{~h}$, collect the non-adherent and migrated NK92MI cells from the bottom chamber and transfer them to fluorescence-activated cell sorting (FACS) tubes for further analysis.

NOTE: The time of culture may vary depending on the type of target cells, as well as the amount and kinetics of chemokines produced by the target cells. Therefore, this time should be empirically determined for each cell type, chemokine, and experiment.

6. Add predetermined number of counting beads for flow cytometry in a volume of $50 \mu \mathrm{L}$ to each tube containing migrated NK cells. Evaluate the volume of $300 \mu \mathrm{L} /$ well cell suspension using any flow cytometer capable of automated FACS-based cell counting.

NOTE: Mix or vortex-mix the counting beads for flow cytometry thoroughly each time before use to ensure that a constant number of beads is used to minimize experimental variability. Reverse pipetting is recommended with count beads to maintain accuracy. Use only NK cells and counting beads for flow cytometry as FACS analysis controls. The authors recommend reading at least 10,000 beads + NK cells combined, an amount that has worked well. However, this number may vary depending upon the experimental conditions. Therefore, the combined number of beads + NK cells should be empirically determined for each type of experiment. Additionally, it is important to perform experiments using biological triplicates to achieve statistically significant results and to account for variability between different cell counts.

7. Calculate absolute number of migrated NK92MI cells using this formula:

Absolute number of migrated NK 92 MI cells $=\frac{A}{B} \times \frac{C}{D}$

where $A=$ number of cells, $B=$ number of beads, $C=$ assigned bead count of the lot (number of counting beads for flow cytometry $/ 50 \mu L$; in this example 49,500$)$, and $D=$ volume of sample $(\mu \mathrm{L})$.

NOTE: If $300 \mu \mathrm{L}$ of sample volume (migrated cells) is used for FACS analysis with $50 \mu \mathrm{L}$ of counting beads for flow cytometry, the absolute number of migrated cells $=1,700$ cells $/ 3,300$ bead events $x 49,500$ beads $/ 300 \mu \mathrm{L}=84.975 \mathrm{cells} / \mu \mathrm{L}$. The calculation should be corrected if the sample is diluted or if a different volume of FACS counting beads are used.

\section{Representative Results}

NK cell cytotoxicity assays and NK cell migration assay were performed using the SK-HEP-1 hepatic tumor cell line as a model system. To measure NK cell cytotoxicity using the LDH assay, SK-HEP-1 cells expressing either a nonspecific (NS) shRNA or shRNA targeting activating transcription factor 4 (ATF4) were incubated with NK92MI cells in a 96 well plate for $3 \mathrm{~h}$ (Figure 1A). ATF4 has been previously shown to regulate NK cell cytotoxicity by upregulating the activating ligand ULBP $1^{28}$. LDH activity associated with NK cell-mediated target cell killing was measured colorimetrically, and percent cytotoxicity calculated using the formula described in protocol 1. ATF4 knockdown significantly reduced NK cell-mediated cytotoxicity compared to NK cells expressing NS shRNA (Figure 1B-D). We also measured NK cell cytotoxicity using a calcein AM staining-based assay. To this end, SK-HEP-1 cells expressing NS shRNA or ATF4-targeting shRNAs were labelled with calcein AM and incubated with NK92Ml cells in 96 well plates for $4 \mathrm{~h}$ as illustrated in Figure 2A. After incubation, images of calcein AM-positive cells were captured by fluorescence microscopy using a FITC/GFP filter. Cells killed by NK cells are not detected by this approach because they no longer retain the calcein AM dye. As shown in Figure 2B,C, the number of calcein AM-positive SK-HEP-1 cells is decreased after co-culture with NK92MI cells compared to SK-HEP-1 cells grown without NK92MI cells. However, as expected, ATF4 knockdown via shRNA reduced NK cellmediated killing of SK-HEP-1 cells, observed by a greater number of calcein AM-positive cells (Figure 2B,C). Therefore, both LDH-based and calcein AM assays showed consistent results and confirmed that ATF4 knockdown reduces NK-mediated cancer cell cytotoxicity. Either of these assays is sufficient to assess NK cell-mediated cytotoxicity; however, we recommend using both methods to increase both the stringency and confidence in the results.

We also present the results of a 24 well NK cell migration assay. NK92MI cells were resuspended in serum-free NK92MI medium in the upper chamber, and chemokine, CC motif, ligand 2 (CCL2) was added to the lower permeable chamber. NK cell migration was assayed as described in section 3 (Figure 3A). The number of migrated NK92MI cells was quantified by adding counting beads for flow cytometry and followed by FACS analysis. As shown in Figure 3B-C, there was a significant increase in the number of NK92MI cells that had migrated toward CCL2-containing medium compared to control medium. 
A

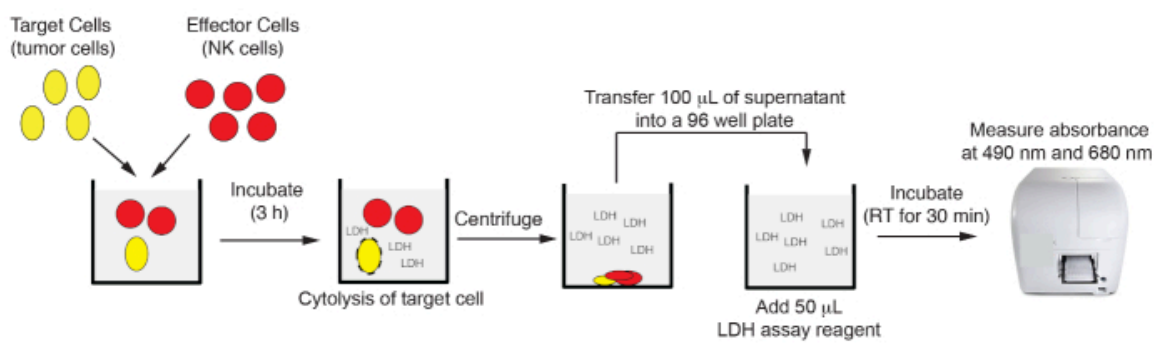

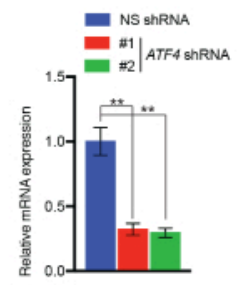

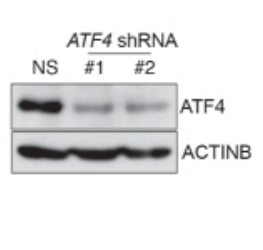

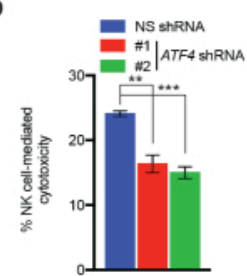

Figure 1: Colorimetric LDH activity-based NK cell-mediated cytotoxicity assay. (A) Schematic depicting the key steps of colorimetric LDH activity-based NK cell cytotoxicity assay. (B,C) ATF4 expression was analyzed in SK-HEP-1 cells expressing either nonspecific (NS) shRNA or shRNAs targeting ATF4 by quantitative RT-PCR and western blotting. (B) Relative ATF4 mRNA level is shown after normalization to ACTINB level in SK-HEP-1 cells expressing NS shRNA or ATF4 shRNAs. (C) ATF4 and ACTINB protein levels in SK-HEP-1 cells expressing NS shRNA or ATF4 shRNAs. (D) NK cell-mediated cytotoxicity was analyzed in SK-HEP-1 cells expressing either NS shRNA or ATF4 shRNAs by the LDH method. Percent (\%) NK cell-mediated cytotoxicity is shown. Data are presented as mean \pm SEM; ns, not significant; ${ }^{* *} p<0.01,{ }^{* * *} p<0.001$. Please click here to view a larger version of this figure. 
A

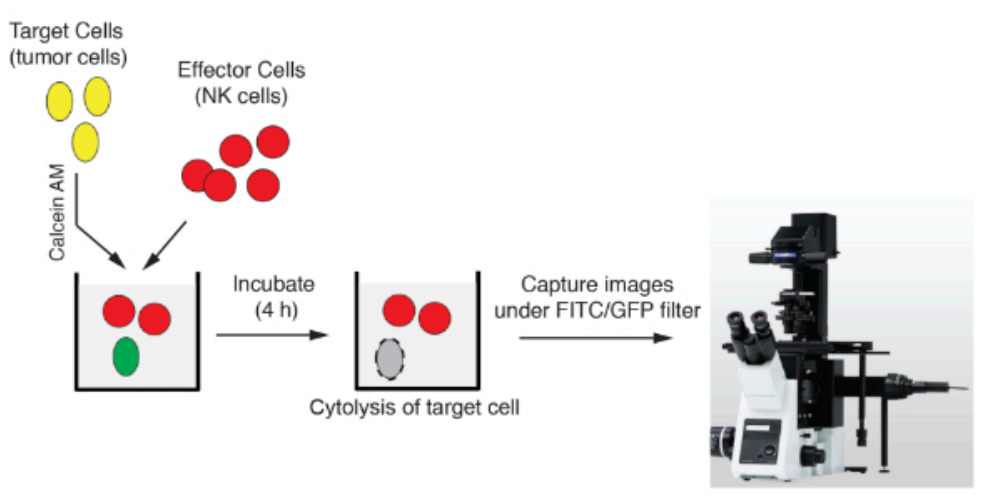

B

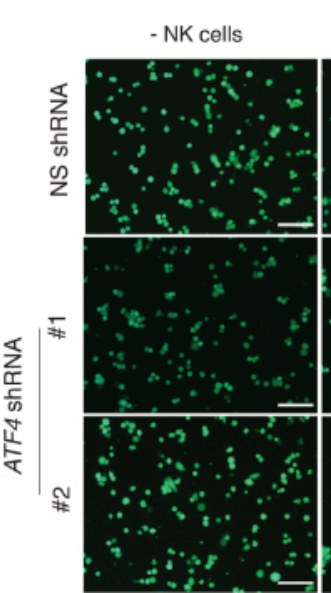

SK-HEP-1

+ NK cells

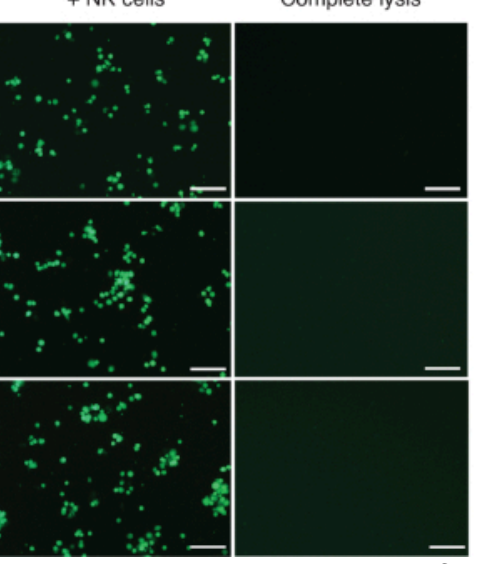
SK-HEP-1

Figure 2: Calcein AM staining-based microscopic method for measuring NK cell-mediated cytotoxicity. (A) Schematic depicting the key steps of calcein AM staining-based microscopic method for measuring NK cell-mediated cytotoxicity. (B) NK cell-mediated cytotoxicity was analyzed in SK-HEP-1 cells expressing either NS shRNA or ATF4 shRNAs using the calcein AM method. Representative images are shown. Scale bar $=200 \mu \mathrm{m}$. (C) Percent of calcein AM-positive cells for the experiment presented in panel B. ${ }^{* *} p<0.01$. Please click here to view a larger version of this figure.

A

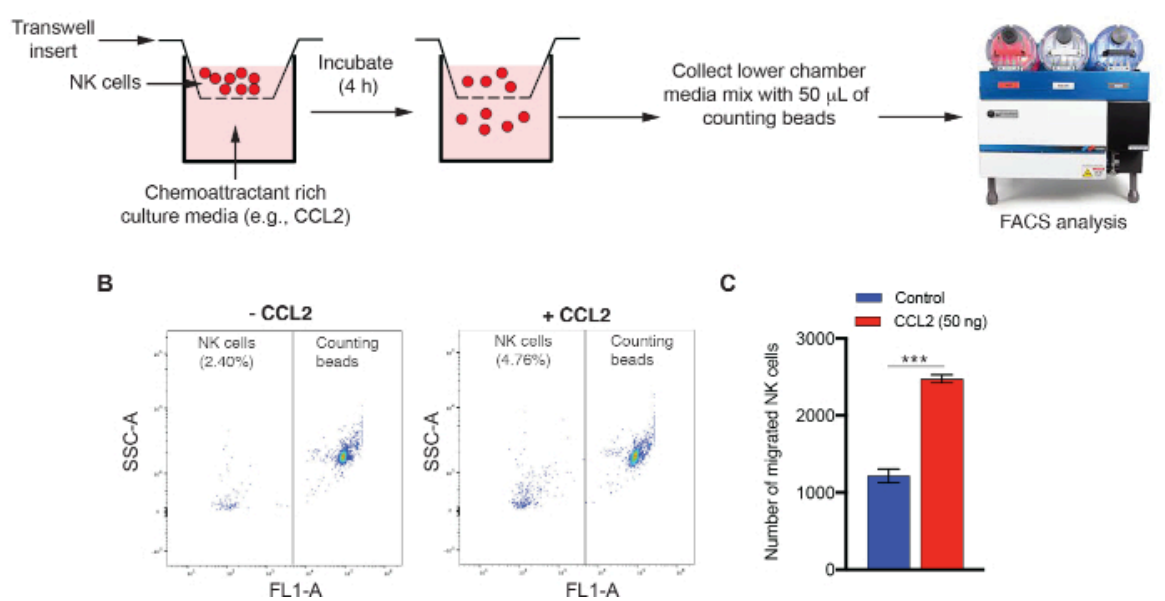

Figure 3: FACS-based quantitative NK cell migration assay. (A) Schematic depicting the key steps of FACS-based NK cell migration assay. $(B, C)$ NK cell migration assay was carried out after adding $50 \mathrm{ng}$ of CCL2 to the bottom chamber of the 24 well plate. (B) Representative NK cell migration dot plots for control or CCL2-treated culture medium are shown. (C) NK cell migration data (mean \pm SEM) is presented for the experiment shown in panel $B .{ }^{* * *} p<0.001$. Please click here to view a larger version of this figure. 


\section{Discussion}

The cytotoxicity and migration methods described here can be used to evaluate NK cell cytotoxicity to cancer cells and NK cell-mediated immune evasion mechanisms in malignant tumors, as well as to identify therapeutic agents that will enhance NK cell activity/function. The protocols are simple, sensitive, reproducible, and preferable alternatives to classical radioactivity-based ${ }^{51}$ chromium release assay. The protocols have been specifically designed to be readily adaptable for use in most laboratories, with straightforward colorimetric, microscopic, or FACS-based readouts that are easy to interpret, allowing researchers to reach reliable conclusions. All are scalable for high-throughput screening-based approaches. Although the protocols are presented in the context of a single hepatic tumor cell line, they can be easily adapted easily to other cancer cell types and/or other non-cancer target cells.

Whereas all of the methods presented are robust and reproducible, inter-experimental variation could occur with different batches of cancer cells and NK cells. To ensure that results accurately support the conclusions of the experiments, it is advisable to repeat the experiment at least two times using biological triplicates.

A limitation of this method is that the growth rate of NK92MI cells can be slow. Therefore, for experiments with 50 or more samples, sufficient numbers of NK92MI should be grown beforehand to prevent delays. Also, all of the controls described in the protocols must be implemented to avoid spurious and nonreproducible results. Another limitation of the NK cytotoxicity assay is that the NK to cancer cell ratio, as well as the incubation time, must be optimized for each target cell. For example, we have tested various ratios of cancer cells to NK cells (1:5, 1:10, 1:20, $1: 40$, and 1:80) for the hepatic cancer cell lines, as well as multiple incubation times $(2,3,4,5$, and $6 \mathrm{~h})$. Based on our results we observed most consistent results with $1: 10$ and 1:20 ratios of cancer cells to NK cells and incubation for $3 \mathrm{~h}$.

In addition, cancer cells derived from solid tumors will attach and grow on the surface of the culture plate, whereas NK cells grow in suspension. If the incubation time is longer than $3 \mathrm{~h}$, it is advisable to use ultralow attachment 96 well plates, which will improve consistency and reproducibility between experiments and biological replicates. It is also important to note that NK cell-induced cytotoxicity assays can also be performed using primary NK cells isolated from peripheral blood mononuclear cells (PBMCs). However, there are several limitations with such experiments. First, these experiments cannot be as easily scaled as with NK92MI cells. Second, batch-to-batch variation of cytotoxic activity of NK cells isolated from PMBCs can be problematic in terms of reproducibility and interpretation of the results. Similarly, other human NK cell lines are described in the literature and could be used in these types of experiments, including NKL cells ${ }^{29}$; however, unlike NK-92MI cells, NKL cells are IL-2 dependent and not commercially available.

When considering the calcein AM experiments described, it is important to note that calcein AM remains in apoptotic bodies after cell death ${ }^{30}$. Therefore, the quantitation of calcein AM staining should be carefully performed, as it may lead to an underestimation of NK cytotoxicity.

Similar to NK cell-mediated cytotoxicity, modulation of NK cell migration by cytokines and other chemoattractants plays an important role in the regulation of NK cell function. The NK cell migration assay described here provides a simple platform to evaluate NK cell migration in the context of a stimulus such as a chemokine or chemoattractant. This assay can be used to evaluate agents that can either promote or interfere with NK cell migration - thus, identifying enhancers and repressors of NK cell migration. This method can also be useful to study the NK cell migration regulatory capacity as a result of a genetic/epigenetic alterations (upregulation or downregulation) or arising due to drug treatment.

To accurately measure NK cell migration, there are few critical steps that should be followed. For all of the experiments using conditioned medium, it is important that equivalent conditioned medium be used from both control and treatment conditions to obtain accurate measurements. The time of incubation will be varied with purified chemoattractants and conditioned medium. Finally, transwell migration assays are well established and considered an excellent method to assess NK cell migration; however, the homogeneous monocultures employed in the assays lack the complex physiology of tissues or even $3 \mathrm{D}$ cultures that might more accurately mimic the tumor microenvironment.

Thus, although there are some limitations to the NK cell cytotoxicity assays and NK migration assay presented in this article, these assays are applicable to a wide range of immunological studies and thus provide important and reliable methods to assess NK cell function and NK cell modulatory immune therapeutics.

\section{Disclosures}

The authors have nothing to disclose.

\section{Acknowledgments}

We gratefully acknowledge grants from the National Institutes of Health: R01CA195077-01A1 (NW), R01CA200919-01 (NW), and 1R01 CA218008-01A1 (NW). We also acknowledge Department of Defense funding W81XWH1910480 and W81XWH-18-1-0069 to NW.

\section{References}

1. Chiossone, L., Dumas, P. Y., Vienne, M., Vivier, E. Natural killer cells and other innate lymphoid cells in cancer. Nature Reviews Immunology. 18 (11), 671-688 (2018).

2. Borghaei, H., Smith, M. R., Campbell, K. S. Immunotherapy of cancer. European Journal of Pharmacology. 625 (1-3), 41-54 (2009).

3. Kalbasi, A., Ribas, A. Tumour-intrinsic resistance to immune checkpoint blockade. Nature Reviews Immunology. (2019).

4. Zhang, Q., Cao, X. Epigenetic regulation of the innate immune response to infection. Nature Reviews Immunology. 19 (7), $417-432$ (2019). 
5. Janeway, C. A., Jr. The immune system evolved to discriminate infectious nonself from noninfectious self. Immunol Today. 13 (1), 11-16 (1992).

6. Natoli, G., Ostuni, R. Adaptation and memory in immune responses. Nature Immunology. 20 (7), 783-792 (2019).

7. Cooper, M. D., Alder, M. N. The evolution of adaptive immune systems. Cell. 124 (4), 815-822 (2006).

8. Riera Romo, M., Perez-Martinez, D., Castillo Ferrer, C. Innate immunity in vertebrates: an overview. Immunology. 148 (2), 125-139 (2016).

9. Sonnenberg, G. F., Hepworth, M. R. Functional interactions between innate lymphoid cells and adaptive immunity. Nature Reviews Immunology. 19 (10), 599-613 (2019).

10. Vivier, E. et al. Innate or adaptive immunity? The example of natural killer cells. Science. 331 (6013), 44-49 (2011).

11. Shi, F. D., Ljunggren, H. G., La Cava, A., Van Kaer, L. Organ-specific features of natural killer cells. Nature Reviews Immunology. 11 (10), 658-671 (2011).

12. Trapani, J. A., Davis, J., Sutton, V. R., Smyth, M. J. Proapoptotic functions of cytotoxic lymphocyte granule constituents in vitro and in vivo. Current Opinion in Immunology. 12 (3), 323-329 (2000).

13. Krzewski, K., Strominger, J. L. The killer's kiss: the many functions of NK cell immunological synapses. Current Opinion in Cell Biology. 20 (5), 597-605 (2008).

14. Berke, G. The binding and lysis of target cells by cytotoxic lymphocytes: molecular and cellular aspects. Annual Review of Immunology. 12, 735-773 (1994).

15. Abel, A. M., Yang, C., Thakar, M. S., Malarkannan, S. Natural Killer Cells: Development, Maturation, and Clinical Utilization. Frontiers in Immunology. 9, 1869 (2018).

16. Zamai, L. et al. Natural killer (NK) cell-mediated cytotoxicity: differential use of TRAIL and Fas ligand by immature and mature primary human NK cells. Journal of Experimental Medicine. 188 (12), 2375-2380 (1998).

17. Marcus, A. et al. Recognition of tumors by the innate immune system and natural killer cells. Advances in Immunology. 122, 91-128 (2014).

18. Vesely, M. D., Kershaw, M. H., Schreiber, R. D., Smyth, M. J. Natural innate and adaptive immunity to cancer. Annual Review of Immunology. 29, 235-271 (2011).

19. Alter, G., Malenfant, J. M., Altfeld, M. CD107a as a functional marker for the identification of natural killer cell activity. Journal of Immunological Methods. 294 (1-2), 15-22 (2004).

20. Bryceson, Y. T. et al. A prospective evaluation of degranulation assays in the rapid diagnosis of familial hemophagocytic syndromes. Blood. 119 (12), 2754-2763 (2012).

21. Shabrish, S., Gupta, M., Madkaikar, M. A Modified NK Cell Degranulation Assay Applicable for Routine Evaluation of NK Cell Function. Journal of Immunology Research. 2016, 3769590 (2016).

22. Pietra, G. et al. Melanoma cells inhibit natural killer cell function by modulating the expression of activating receptors and cytolytic activity. Cancer Research. 72 (6), 1407-1415 (2012).

23. Fehniger, T. A. et al. Potential mechanisms of human natural killer cell expansion in vivo during low-dose IL-2 therapy. Journal of Clinical Investigation. 106 (1), 117-124 (2000).

24. Brunner, K. T., Mauel, J., Cerottini, J. C., Chapuis, B. Quantitative assay of the lytic action of immune lymphoid cells on 51-Cr-labelled allogeneic target cells in vitro; inhibition by isoantibody and by drugs. Immunology. 14 (2), 181-196 (1968).

25. Roder, J. C. et al. A new immunodeficiency disorder in humans involving NK cells. Nature. 284 (5756), $553-555$ (1980)

26. Byrd, A., Hoffmann, S. C., Jarahian, M., Momburg, F., Watzl, C. Expression analysis of the ligands for the Natural Killer cell receptors NKp30 and NKp44. PLoS One. 2 (12), e1339 (2007).

27. Nitahara, A. et al. NKG2D ligation without T cell receptor engagement triggers both cytotoxicity and cytokine production in dendritic epidermal T cells. Journal of Investigative Dermatology. 126 (5), 1052-1058 (2006).

28. Gowen, B. G. et al. A forward genetic screen reveals novel independent regulators of ULBP1, an activating ligand for natural killer cells. Elife. 4, e08474 (2015).

29. Robertson, M. J. et al. Characterization of a cell line, NKL, derived from an aggressive human natural killer cell leukemia. Experimental Hematology. 24 (3), 406-415 (1996).

30. Somanchi, S. S., McCulley, K. J., Somanchi, A., Chan, L. L., Lee, D. A. A Novel Method for Assessment of Natural Killer Cell Cytotoxicity Using Image Cytometry. PLoS One. 10 (10), e0141074 (2015) 